\title{
ArcheoSciences
}

Revue d'archéométrie

\section{Identifier et enregistrer des vestiges archéologiques sous couvert forestier à partir de données LiDAR : méthode et limites}

Identify and record archaeological remains under forests using LiDAR data: method and limits

Emmanuel Chevigny, Laure Saligny, Ludovic Granjon, Dominique Goguey, Alexandra Cordier, Yves Pautrat et Alain Giosa

\section{OpenEdition}

Journals

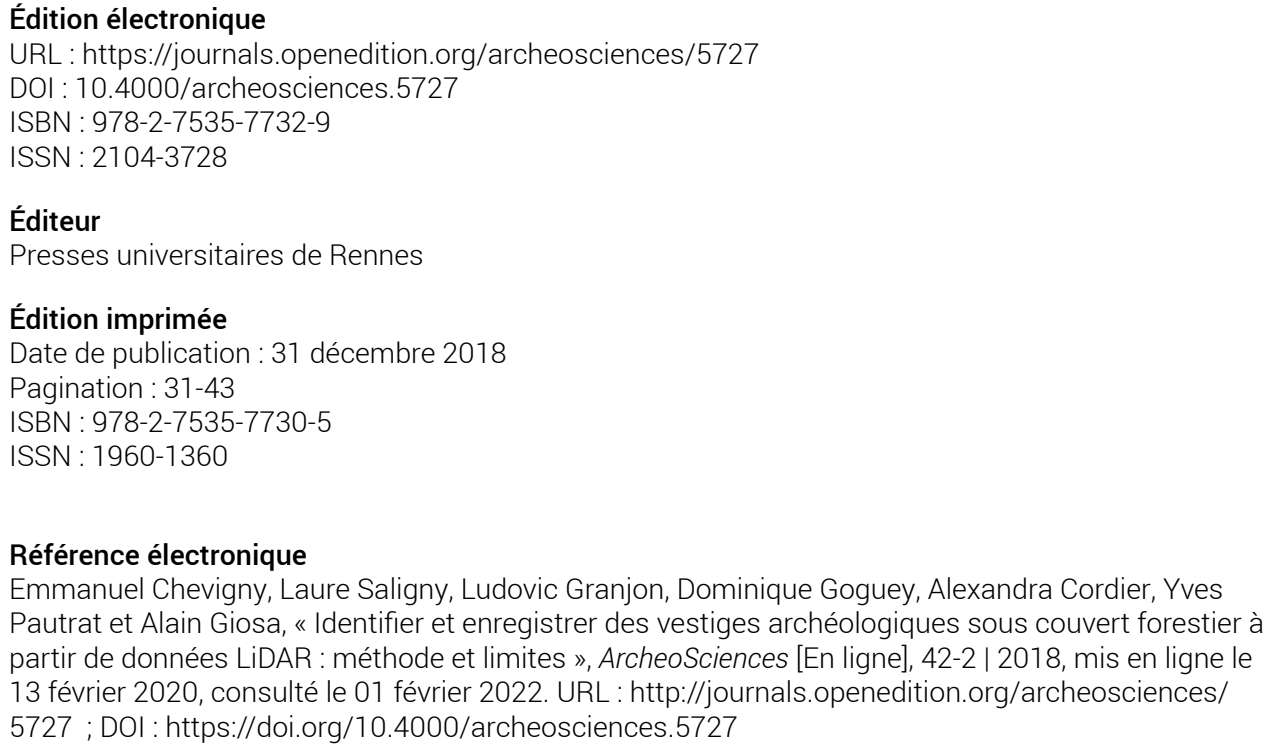




\title{
Identifier et enregistrer des vestiges archéologiques sous couvert forestier à partir de données LiDAR : méthode et limites
}

\author{
Identify and Record Archaeological Remains under Forests Using LiDAR Data: \\ Method and Limits
}

\author{
Emmanuel ChevignYa, Laure SALIGNY ${ }^{a}$, Ludovic Granjon ${ }^{a}$, \\ Dominique Goguer ${ }^{\mathrm{b}}$, Alexandra Cordier ${ }^{\mathrm{b}}$, Yves Pautrat ${ }^{\mathrm{c}}$ et Alain Giosa ${ }^{\mathrm{d}}$
}

\begin{abstract}
Résumé : Depuis plus de dix ans, des recherches sur les ensembles de structures en pierre sous forêt sont menées dans la forêt du Châtillonnais (Côte-d'Or, France). Les prospections réalisées jusqu’à ce jour consistaient en un relevé GNSS systématique des structures. Ces prospections ont été complétées en 2012 par une acquisition LiDAR sur $400 \mathrm{~km}^{2}$ réalisée par les Parcs Nationaux de France (PNF). Cet article a pour objectif de présenter la démarche développée au sein du projet, pour traiter et enregistrer les données archéologiques détectées par le LiDAR. L'enjeu de celle-ci est d'harmoniser la saisie et les modalités d'enregistrement des structures identifiées (à partir des jeux d'images dérivées des données LiDAR) par les différents chercheurs. Pour la mise en place de cette méthode, un test de vectorisation a été réalisé sur un même secteur par huit chercheurs afin d'identifier les biais de vectorisation puis de proposer un processus méthodologique associant les indices de visualisation utilisés, la nature des vestiges à détecter et leur implantation topographique et des techniques d'enregistrement (base de données spatiales, outils de vectorisation, etc.) connectées aux méthodes de relevés de terrain.
\end{abstract}

\begin{abstract}
Since more than ten years, researches about stone remains under forest have been carried out in the Châtillonnais (Côte-d'Or, France). The surveys carried out up to date consisted of a systematic GNSS survey of the archaeological features. These surveys were completed in 2012 by a LiDAR acquisition over $400 \mathrm{~km}^{2}$, funded by Parcs Nationaux de France (PNF). This paper aims to present the approach developed within the project to process and record the archaeological data detected with the LiDAR. The challenge is to homogenize the vectorization and the recording of each identified structures (from the sets of images derived from the LiDAR data) by the different researchers. For the implementation of this method, a test of vectorization was realized on the same geographical area by eight researchers. The aim of this test is to identify the vectorization biases and to propose a methodological process associating the visualization indexes used, the type of remains to be detected and their topographic location and recording methodology (spatial database, tools of vectorization, etc.) connected to the survey methodology.
\end{abstract}

Mots clés : LiDAR, MNT, entité archéologique, bases de données spatiales, méthodes de vectorisation.

Keywords: Lidar, DEM, archaeology feature, spatial database vectorization.

${ }^{a}$ USR CNRS - UBFC 3516, plateforme technologique GEOBFC, 6 esplanade Erasme, F-21066 DIJON Cedex. (chevigny.emmanuel@gmail.com) (laure. saligny@u-bourgogne.fr) (ludovic.granjon@u-bourgogne.fr)

b UMR CNRS - UBFC 6298 ArTeHiS, 6 boulevard Gabriel, F-21000 DIJON. (dominique.goguey@orange.fr) (alexandra.cordier@u-bourgogne.fr)

' DRAC de Bourgogne, SRA, 39-41 rue Vannerie, F-21000 DIJON. (yves.pautrat@culture.gouv.fr)

d UMR 7041 ArScAn - Archéologies environnementales, Maison de l'Archéologie et de l'Ethnologie, 21 allée de l'Université, F-92023 NANTERRE Cedex.

(alain.giosa@mae.univ-paris1.fr) 


\section{INTRODUCTION}

Depuis plus d'une dizaine d'années, la technologie LiDAR (light detection and ranging) est utilisée dans le domaine de la prospection aéroportée appliquée à l'archéologie (Nuninger et al., 2010). Cette technologie produit des mesures à haute précision et haute densité de la topographie de la surface du sol. Le LiDAR présente un intérêt certain en archéologie sous couvert forestier, puisqu'il permet l'identification de vestiges archéologiques formant des microreliefs. L'utilisation du LiDAR en archéologie est en plein essor et touche l'ensemble de la communauté archéologique i.e. en France (Sittler et Hauger, 2007; Georges-Leroy et al., 2009), en Europe (Kooistra et Maas, 2008; Bofinger et Hesse, 2010), voire mondiale (Chase et al., 2010; Evans, 2016). Toutes ces études ont démontré le potentiel du LiDAR pour la détection de structures archéologiques sous couvert forestier. De plus, cette technologie permet une investigation pour des surfaces importantes (de l'ordre de plusieurs centaines de kilomètres carrés), dans un laps de temps court, à une résolution spatiale élevée (de l'ordre du décimètre), et une très bonne précision (de l'ordre du centimètre) (Devereux et al., 2005).

La détection de vestiges archéologiques est habituellement réalisée à partir de techniques de visualisation du Modèle Numérique de Terrain (MNT) dérivé des données LiDAR. Ces techniques permettent de localiser et de proposer une interprétation archéologique des structures observées. Depuis l'utilisation du LiDAR en archéologie, l'ombrage (billshading) a été très largement utilisé, car il est facile à calculer et à interpréter (Zakšek et al., 2011). Cependant l'utilisation de l'ombrage comme technique principale de reconnaissance peut avoir un impact négatif sur le nombre de structures identifiées et sur la qualité de leur interprétation (Devereux et al., 2005, 2008). L'utilisation de l'analyse en composantes principales (ACP) sur plusieurs ombrages permet de synthétiser l'information en réduisant le nombre de bandes. Généralement, les trois premières bandes de l'ACP renferment plus de $99 \%$ de la variabilité du système (Zakšek et al., 2011). Des techniques plus sophistiquées ont été développées pour pallier aux problèmes rencontrés sur l'ombrage. Parmi celles-ci, le sky view factor (Kokalj et al., 2011; Zakšek et al., 2011), l'ouverture topographique (Doneus, 2013; Yokoyama et al., 2002) et les cartes de radiation solaire permettent d'observer le degré de dominance ou de fermeture d'une zone sur une surface irrégulière. Le modèle de relief local (Local Relief Model «LRM ») permet quant à lui de représenter les différences d'altitudes locales observées à grandes échelles, après avoir supprimé la morphologie du paysage observée à petites échelles, à partir d'un MNT (Hesse, 2010). On peut alors voir si la structure présente un relief positif ou négatif et ainsi évaluer sa hauteur ou sa profondeur. Enfin, d'autres techniques, telles que les cartes des pentes, d'orientation, de courbure (Doneus et Briese, 2006) permettent d'effectuer une classification des structures selon leurs caractéristiques morphologiques.

L'utilisation d'une seule technique de visualisation ne permet pas forcement d'identifier toutes les structures (Challis et al., 2011). La combinaison de plusieurs techniques peut, dans certains cas, augmenter les contrastes tout en supprimant le bruit produit par certaines techniques (Hesse, 2010). Le contexte géomorphologique i.e. plateau, versant, plaine alluviale, peut également avoir un impact sur la reconnaissance des structures, certaines techniques permettent de mieux discriminer les structures que d'autres (Stular et al., 2012). De plus, les structures présentent des morphologies qui leurs sont propres; le choix des paramètres utilisés pour le calcul d'indice doit être bien défini en fonction du type de structure recherchée (Hesse, 2010; Nuninger et al., 2010; Zakšek et al., 2011). Toutes ces techniques de visualisation permettent de localiser les vestiges archéologiques, cependant elles ne permettent pas forcement d'aboutir à une caractérisation typologique des structures.

Cette étude présente les travaux méthodologiques effectués au sein de la plateforme technologique géomatique de Bourgogne Franche-Comté de la MSH de Dijon (GEOBFC - MSH de Dijon) sur la détection et l'enregistrement de structures archéologiques sous couvert forestier à partir de données LiDAR. Ces travaux ont été réalisés sur les données LiDAR de la forêt du Châtillonnais, acquises par Parcs Nationaux de France pour le groupement d'intérêt public (GIP) des forêts de Champagne et Bourgogne. En collaboration avec les archéologues qui étudient le secteur, une démarche de vectorisation a été mise en place, d'une part pour accompagner les archéologues qui utilisaient pour la première fois des données $\mathrm{LiDAR}$ et d'autre part pour aboutir à une vectorisation la plus homogène et cohérente possible de l'ensemble du secteur investigué. Dans un premier temps, une méthode de reconnaissance des structures archéologiques selon une typologie morphologique a été développée à partir de divers indices de visualisation. Puis, la mise en place d'un Système d'Information Géographique (SIG) comprenant un protocole de vectorisation, une base de données spatiales, les données nécessaires à l'identification des structures et le paramétrage du logiciel libre QGIS a permis de proposer une solution adaptée à la reconnaissance et à l'enregistrement des structures. Enfin, cette solution a été testée par divers opérateurs sur un secteur commun afin d'évaluer l'homogénéité de la vectorisation. Ce test a conduit d'une part à améliorer le protocole de vectorisa- 
tion et d'autre part à identifier les facteurs qui contribuent à rendre la vectorisation hétérogène et l'identification des structures archéologiques incomplète voire erronée.

\section{ConteXte de l'Étude}

\section{La forêt du Châtillonnais et son LiDAR}

Le secteur d'étude s'étend sur une partie de la forêt domaniale de Châtillon-sur-Seine (Côte d'Or, France) dans le futur Parc national des forêts de Champagne et Bourgogne (fig. 1). Les forêts du Châtillonnais ont fait l'objet de nombreuses recherches archéologiques depuis le $\mathrm{XIX}^{\mathrm{e}}$ siècle jusqu’à nos jours (Paris et Bretonne, 1981; Chaume et Mordant, 2011). Depuis plus de dix ans, un inventaire exhaustif des structures de pierres sèches a été mis en œuvre par des prospections pédestres au GNSS différentiel et complété par des prospections de terrain (Goguey et al., 2010). Pour compléter ces travaux, une acquisition LiDAR a été réalisée en mars 2012, par le GIP du futur Parc national des forêts de Champagne et Bourgogne. L'acquisition réalisée en trois jours, en mars 2012, par la société Sintegra (Grenoble) couvre un secteur de $381 \mathrm{~km}^{2}$, pour une densité moyenne de points "sol" égale à 7 points $/ \mathrm{m}^{2}$. La classification des points «sol/sursol " a été réalisée par la société chargée de l'acquisition. Le filtrage utilisé pour classifier les données a été discuté avec les archéologues, sur des secteurs déjà pros- pectés, pour préserver le maximum d'informations archéologiques à l'issue de la classification. Un modèle numérique de terrain (MNT) à une résolution spatiale de $50 \mathrm{~cm}$ a été calculé par la même société à partir des points « sols » estimés par la classification.

\section{Typologie des structures basée sur la morphologie des structures}

Depuis plus de 10 ans, des campagnes de relevés, effectuées à l'aide de GNSS différentiels, ont été réalisées sur le secteur d'étude. Pour réaliser ces relevés, un thésaurus des structures visibles sur le terrain a été formalisé, thésaurus fondé sur la morphologie des vestiges (murées, fossés, tertres, excavations, etc.) (Goguey et al., 2010, Chaume et Mordant, 2011). La typologie utilisée pour ces relevés définit trois formes de structures principales i.e. des lignes, des points, des surfaces. Pour chacune de ces formes, les structures présentent soit un relief négatif, soit un relief positif, soit un relief de rupture de pente. La taille de la structure et sa position géomorphologique ont également été prises en compte. Dans un second temps, la cartographie de ces formes simples et l'étude de leurs relations spatiales ont permis d'identifier et de caractériser des structures dites « complexes ", tels que des parcellaires, des enclos, des habitats, etc. Pour certains vestiges, la fonction usuelle a pu être déterminée uniquement à partir de fouilles, tels que les tertres

Figure 1 : Emprise du vol LiDAR réalisé, d'une superficie de $380 \mathrm{~km}^{2}$, sur la forêt du Châtillonnais.

Figure 1: Area of the LiDAR flight on the Châtillonnais forest $\left(380 \mathrm{~km}^{2}\right)$.

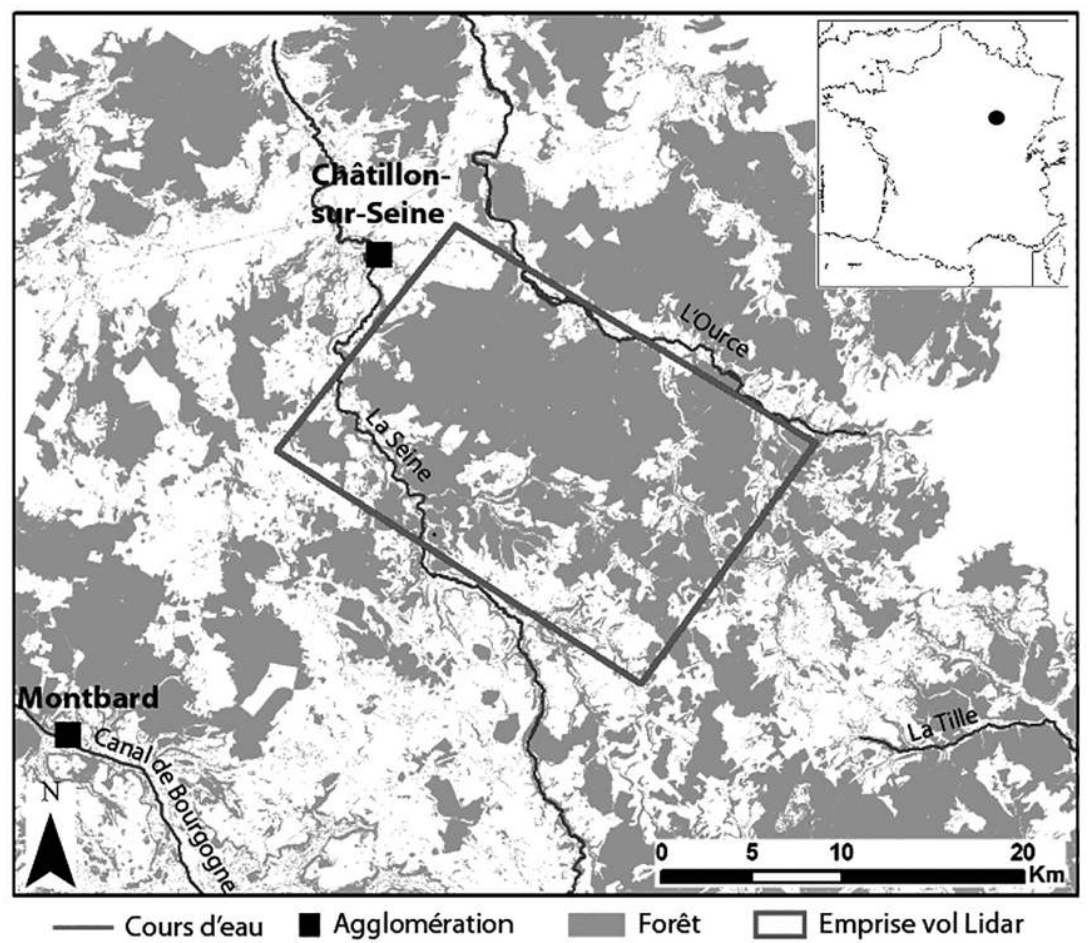


tumulaires, les habitats etc. (Goguey et al., 2010). Cette typologie étant basée sur les paramètres morphologiques, l'utilisation de données topographiques à haute résolution spatiale, comme les données LiDAR, semble être adaptée à l'identification et la caractérisation des vestiges archéologiques. Cette démarche a également été employée dans la forêt de Haye, en Lorraine (Georges-Leroy et al., 2011) ou dans la forêt de Chailluz, près de Besançon (Fruchart, 2014) dans des contextes environnementaux similaires à celui du Châtillonnais.

\section{Mise EN GEUVRE D'UN PROTOCOLE DE VECTORISATION}

Ce protocole pour la reconnaissance des vestiges, pour leur enregistrement et pour leur interprétation archéologique a été développé au sein de la plateforme technologique GEOBFC à la Maison des Sciences de l'Homme de Dijon. Ces travaux se sont déroulés en trois étapes. Tout d'abord une première étape a concerné l'identification des types de structures à partir des données LiDAR. La deuxième étape a permis la rédaction d'un modus operandi de vectorisation et la création d'une application SIG pour la vectorisation et l'enregistrement des structures. La finalité de cette démarche et de cette application est d'accompagner les chercheurs dans le traitement des données LiDAR et d'obtenir une vectorisation la plus homogène entre les différents chercheurs et opérateurs travaillant à partir du LiDAR de la forêt du Châtillonnais. Enfin, nous avons soldé la mise en œuvre par un test collectif de reconnaissance et d'enregistrement des vestiges archéologiques sur un secteur commun afin de valider la démarche et l'application.

\section{Identification et enregistrement des structures}

\section{Identification du type de structures}

La typologie utilisée pour les relevés GNSS, définie selon des critères morphologiques, a été adaptée à l'analyse et à l'interprétation des données LiDAR. Pour tester la reconnaissance typologique des structures, les techniques de visualisations du MNT précédemment introduites ont été utilisées. Les relevés GNSS acquis au cours de dix dernières années ont été utilisés pour identifier les anomalies de relief observées sur les divers indices produits à partir du MNT. Parmi ces indices, l'indice des pentes a permis de mettre en évidence les variations morphologiques des structures et ainsi d'identifier leur type. Elle exprime pour chaque pixel du MNT l'inclinaison de la surface topographique mesu- rée par rapport à l'horizontale (Jones, 1998). Les valeurs calculées sont exprimées en degrés $\left(^{\circ}\right)$. Des clés de lecture à partir de cette carte des pentes, ont été proposées permettant ainsi l'identification de chaque type d'anomalie (fig. 2). Par exemple, une murée se caractérise sur la carte des pentes par une succession de valeurs de pente modérées, puis faibles, puis à nouveau modérées. Cette succession permet de distinguer les flancs des murées, correspondant aux valeurs de pentes modérées, du sommet de la murée, correspondant aux faibles valeurs de pente. L'ensemble des structures relevées au GNSS a pu ainsi être caractérisé à partir de cet indice. Toutefois, certaines structures présentent un motif similaire sur la carte des pentes, c'est le cas entre les murées et les fossés, ou bien encore entre les tertres (tas de pierres) et les excavations (minières, lavières). La carte des pentes ne permet par ailleurs pas de faire la distinction entre un relief positif et négatif (Doneus, 2006). Aussi, pour s'affranchir de ce problème, un second indice a été utilisé : l'ouverture topographique positive. Cet indice exprime le degré de dominance ou de fermeture d'un espace sur une surface irrégulière (Doneus, 2013). L'ouverture topographique étant calculée sur des angles importants, supérieurs à $180^{\circ}$, elle permet de s'affranchir des effets topographiques. On peut alors distinguer les structures en élévation des structures en dépression qui ne peuvent être différentiées sur la carte des pentes (fig. 2).

\section{L'enregistrement des structures}

Afin d'homogénéiser l'enregistrement de l'ensemble des données, un Système d'Information Géographique (SIG) a été réalisé. Pour cela, un protocole de vectorisation a été mis au point en collaboration avec les chercheurs en prenant en compte les clés de lecture vues précédemment. L'enregistrement des anomalies s'effectue par le biais du logiciel libre QGIS. Dans ce SIG, les indices de pentes et d'ouverture topographique positive nécessaires à l'identification des structures ont été intégrés. Afin d'affiner et de permettre l'identification d'objets non prévus dans la clé d'identification initiale, d'autres indices ont été intégrés : sky view factor, ouverture topographique négative, 8 ombrages, ACP sur ombrages. Ces différents indices ont été générés par le géomaticien en charge du projet en adaptant les paramètres aux objets à détecter, puis mis à disposition des différents opérateurs.

Les données vectorisées sont intégrées au sein d'une base de données spatiale (SQLITE/Spatialite). La vectorisation des structures s'effectue en deux temps. Tout d'abord, les structures "simples" (vectorisation 1), identifiées comme des objets isolés et d'orientations différentes sont vectorisées (fig. 3A). Les objets sont vectorisés selon trois formes i.e. des 


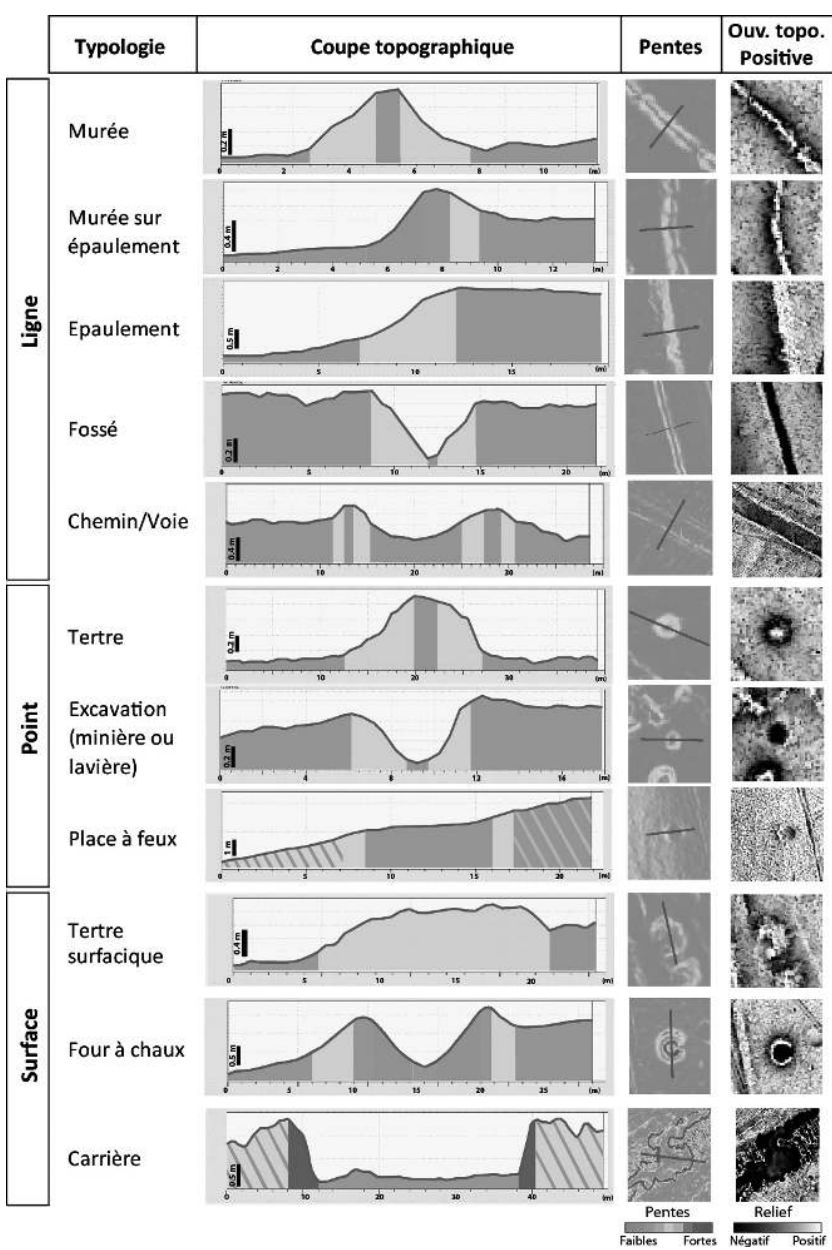

Figure 2 : (Voir planche couleur IX) Grille de lecture de structures archéologiques sur images de pentes et d'ouverture topographique positive.

Figure 2: (See colour plate IX) Reading grid of archaeological remains on slope and openess.

points, des lignes et des polygones et rattachés à trois tables attributaires distinctes.

La saisie du contenu des tables de ce premier niveau d'identification peut se faire en plusieurs étapes. En effet, les attributs peuvent être regroupés en deux catégories, une catégorie descriptive : "qu'est-ce que j'observe sur les images? Quelles sont les formes que j'identifie? »; une seconde plus interprétative concerne les hypothèses des archéologues ou est complétée après prospection ou sondages. Cette seconde catégorie ne sera pas traitée dans le cadre de cet article.

Les attributs renseignés au sein de chaque table sont :

- le type d'indice utilisé pour identifier la structure;

- un identifiant de structure unique, composé des initiales de l'opérateur suivi d'une numérotation incrémentée automatiquement;
- le type de structure identifiée;

- sa fonction;

- l'« incertitude " pour permettre à l'opérateur d'exprimer le degré de fiabilité qu'il estime concernant la reconnaissance du type de structure;

- le champ "vérification terrain " permet de valider le type de structure identifié si celui-ci a été observé par la suite sur le terrain.

Dans un second temps, la vectorisation des structures dites " complexes" (vectorisation 2, fig 3B), formées par plusieurs structures simples, est effectuée. Cette vectorisation correspond à l'interprétation archéologique des structures observées. Ces dernières sont enregistrées sous forme de polygone, au sein d'une quatrième table, dans laquelle sont renseignés le type et la fonction. Ce regroupement des formes simples des vestiges identifiés et interprétés initialement est utile pour étudier des espaces à une échelle plus petite, pour comparer des secteurs et pour certaines analyses spatiales et morphologiques (non abordées dans cet article).

Pour faciliter la saisie de l'ensemble des structures et éviter les erreurs de frappe qui pourraient nuire aux requêtes spatiales, la saisie des divers attributs s'effectue à partir de formulaires de saisie. Afin d'homogénéiser l'enregistrement des structures, des règles de vectorisation ont été utilisées, celles-ci sont en partie basées sur les normes cartographiques proposées par Murielle Georges-Leroy (Georges-Leroy et al., 2011). À titre d'exemple, pour les structures linéaires, l'enregistrement de deux structures distinctes se fait lorsque l'angle formé entre les deux linéaires est supérieur à $45^{\circ}$.

\section{Le test de vectorisation}

Lobjet de ce test consiste à vectoriser un secteur d'un kilomètre carré à l'aide de l'application SIG et du protocole de vectorisation présentés précédemment. La reconnaissance des structures peut se faire à partir de l'ensemble des indices disponibles mais l'opérateur doit saisir celui qui l'a le plus aidé pour l'identification. Pour rappel, les différents indices ont le même paramétrage pour l'ensemble des opérateurs. Par ailleurs, la vectorisation doit être exhaustive pour l'ensemble des objets décrits dans la typologie.

Le secteur choisi pour le test combine différents contextes géomorphologiques (plateau, versant, fond de vallée) et la majeure partie des types de structures étudiées. Ce secteur est représentatif de l'environnement de la forêt du Châtillonnais et à l'avantage d'avoir été prospecté en 2003 avec enregistrements des structures via GNSS.

Le test de vectorisation a été réalisé par huit opérateurs qui présentent des profils divers par leurs fonctions professionnelles et leurs domaines de compétences (archéologues 


\begin{tabular}{|c|c|c|c|c|c|c|c|c|c|c|c|}
\hline \multicolumn{12}{|c|}{ Vectorisation 1} \\
\hline \multicolumn{3}{|c|}{ Divers } & \multicolumn{5}{|c|}{ Identification structure } & \multicolumn{4}{|c|}{ Compléments archéologiques } \\
\hline $\begin{array}{r}\text { Identifiant } \\
\text { structure }\end{array}$ & Date & \begin{tabular}{|l} 
Identifiant \\
Raster utilisé
\end{tabular} & Forme & Relief & Géométrie & Structure & Incertitude & Fonction/Domaine & Datation & $\begin{array}{l}\text { Vérif. } \\
\text { terrain }\end{array}$ & Notes \\
\hline \multirow{3}{*}{$\begin{array}{l}A \\
u \\
t \\
o \\
m \\
a \\
t \\
i \\
q \\
u \\
e\end{array}$} & \multirow{3}{*}{$\begin{array}{l}A \\
u \\
t \\
o \\
m \\
a \\
t \\
i \\
q \\
u \\
e\end{array}$} & \multirow{3}{*}{$\begin{array}{c}\text { SVF } \\
\text { Pentes } \\
\text { OuvTopoPos } \\
\text { OuvTopoNeg } \\
\text { ACP_Omb } \\
\text { Ombrage } \\
\text { MNT }\end{array}$} & 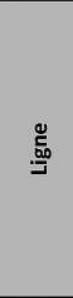 & $\begin{array}{c}\text { Positif } \\
\text { Négatif } \\
\text { Rupture de pente }\end{array}$ & $\begin{array}{l}\text { Courbe } \\
\text { Droite } \\
\text { Irrégulière }\end{array}$ & \begin{tabular}{|c|} 
Indéterminée \\
Affleurement géologique \\
Cours d'eau \\
Epaulement \\
Fossé \\
Murée/levée \\
Murée/levée sur épaulement \\
Muret \\
Ravine \\
Tertre \\
Voie
\end{tabular} & $\begin{array}{c}\text { Incertain } \\
\text { Hésitant } \\
\text { Sûr et certain }\end{array}$ & \begin{tabular}{|c} 
Défensif \\
Extraction \\
Géologique \\
Hydraulique \\
Limite de terrasse \\
Limite parcellaire \\
Voie communication
\end{tabular} & $\begin{array}{c}\text { Antique } \\
\text { Contemporaine } \\
\text { Médiévale } \\
\text { Moderne } \\
\text { Préhistorique } \\
\text { Proto/antique } \\
\text { Protohistorique }\end{array}$ & $\begin{array}{l}\text { oui } \\
\text { non }\end{array}$ & Champ libre \\
\hline & & & 䓂 & $\begin{array}{c}\text { Positif } \\
\text { Négatif } \\
\text { Rupture de pente }\end{array}$ & \begin{tabular}{|c} 
Circulaire \\
Irrégulière angulaire \\
Irrégulière lobée \\
Ovoïde \\
Quadrangualaire
\end{tabular} & $\begin{array}{l}\text { Indéterminée } \\
\text { Carrière } \\
\text { Charbonnière } \\
\text { Déblais } \\
\text { Four à chaux } \\
\text { Minière } \\
\text { Tertre }\end{array}$ & \begin{tabular}{|c||} 
Incertain \\
Hésitant \\
Sûr et certain
\end{tabular} & \begin{tabular}{|c} 
Artisanat/Industrie \\
Extraction \\
Funéraire \\
Limite parcellaire
\end{tabular} & $\begin{array}{c}\text { Antique } \\
\text { Contemporaine } \\
\text { Médiévale } \\
\text { Modeme } \\
\text { Préhistorique } \\
\text { Proto/antique } \\
\text { Protohistorique }\end{array}$ & $\begin{array}{l}\text { oui } \\
\text { non }\end{array}$ & Champ libre \\
\hline & & & 苋 & $\begin{array}{c}\text { Positif } \\
\text { Négatif } \\
\text { Rupture de pente }\end{array}$ & \begin{tabular}{|} 
Circulaire \\
Irrégulière angulaire \\
Irrégulière lobée \\
Ovoïde \\
Quadrangualaire
\end{tabular} & $\begin{array}{l}\text { Indéterminée } \\
\text { Batiment } \\
\text { Carrière } \\
\text { Déblais } \\
\text { Lapiaz } \\
\text { Paléochenal } \\
\text { Tertre } \\
\text { Traces agricoles }\end{array}$ & \begin{tabular}{|c||} 
Incertain \\
Hésitant \\
Sûr et certain
\end{tabular} & \begin{tabular}{|c} 
Agricole \\
Artisanat/Industrie \\
Défensif \\
Extraction \\
Funéraire \\
Habitat \\
Limite parcellaire
\end{tabular} & $\begin{array}{l}\text { Antique } \\
\text { Contemporaine } \\
\text { Médiévale } \\
\text { Modeme } \\
\text { Préhistorique } \\
\text { Proto/antique } \\
\text { Protohistorique }\end{array}$ & $\begin{array}{l}\text { oui } \\
\text { non }\end{array}$ & Champ libre \\
\hline
\end{tabular}

Figure 3A : Structuration de la base de données géographiques intégrant la première étape de vectorisation. Figure 3A: Geographical database for the first vectorization.

\begin{tabular}{|c|c|c|c|c|c|c|}
\hline \multicolumn{7}{|c|}{ Vectorisation 2} \\
\hline $\begin{array}{l}\text { Identifiant } \\
\text { structure }\end{array}$ & Forme & Structure & Fonction/Domaine & Incertitude & Notes & Date \\
\hline 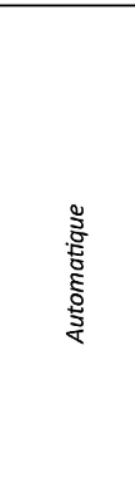 & 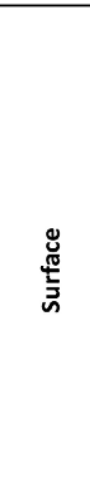 & \begin{tabular}{|c|} 
Parcelle fermée \\
Parcelle ouverte \\
Terrasse \\
Enclos fermé \\
Enclos ouvert \\
Groupement de tertres \\
Groupement de terrasses \\
Groupement de charbonnières \\
Bâtiment \\
Champ bombé \\
Cabane de charbonnier \\
Tumulus \\
Tumulus fouillé \\
Eperon barré/enceinte
\end{tabular} & $\begin{array}{c}\text { Agricole } \\
\text { Artisanat/Industrie } \\
\text { Habitat } \\
\text { Cultuel } \\
\text { Funéraire } \\
\text { Défensif }\end{array}$ & $\begin{array}{c}\text { Incertain } \\
\text { Hésitant } \\
\text { Sûr et certain }\end{array}$ & 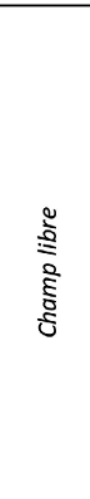 & 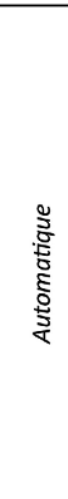 \\
\hline
\end{tabular}

Figure 3B : Structuration de la base de données géographiques intégrant la seconde étape de vectorisation.

Figure 3B: Geographical database for the second vectorization. professionnels, doctorants en archéologie et géomaticiens, tabl. 1). Les archéologues ont de très bonnes connaissances du terrain et des vestiges après plus de dix ans de prospections pédestres sur le secteur d'étude; ils sont néanmoins novices en ce qui concerne l'utilisation des logiciels SIG et le traitement des données LiDAR. Les doctorants archéologues ont des connaissances plus faibles sur l'archéologie forestière des lieux mais ils connaissent les outils SIG. Enfin les ingénieurs géomaticiens sont familiarisés avec les logiciels SIG et le traitement des données LiDAR; leurs connaissances en archéologie forestière sont peu développées.
Les résultats des différents opérateurs ont été comparés à la vectorisation du géomaticien en charge du projet, à la fois expert des Systèmes d'Information Géographiques et des relevés terrain sur le secteur. Les doutes éventuels seront levés par un retour terrain.

Dans un deuxième temps, ce secteur d'étude a été comparé aux relevés GNSS existants sur le secteur pour évaluer un éventuel apport quantitatif et/ou qualitatif du LiDAR. 


\begin{tabular}{|c|c|c|c|c|}
\hline \multirow[b]{2}{*}{ Référence opérateur } & \multirow[b]{2}{*}{ Fonction opérateur } & \multicolumn{3}{|c|}{ Compétences et connaissances } \\
\hline & & Archéologie forestière & $\begin{array}{l}\text { Relevés GPS } \\
\text { forêt Châtillon }\end{array}$ & SIG / traitement Lidar \\
\hline Opérateur 1 & Doctorant archéologie & ++ & + & + \\
\hline Opérateur 2 & Doctorant archéologie & +++ & +++ & + \\
\hline Opérateur 3 & Archéologue & +++ & +++ & $\mathrm{O}$ \\
\hline Opérateur 4 & Archéologue & +++ & +++ & $\mathrm{O}$ \\
\hline Opérateur 5 & Géomaticien & + & ++ & +++ \\
\hline Opérateur 6 & Géomaticien & $\mathrm{O}$ & $\mathrm{O}$ & +++ \\
\hline Opérateur 7 & Géomaticien & + & ++ & ++ \\
\hline Opérateur 8 & Archéologue & +++ & +++ & $\mathrm{O}$ \\
\hline
\end{tabular}

Tableau 1 : Profils des divers opérateurs ayant participés au test de vectorisation. Table 1: Scientific domains of each operator participating to the vectorisation text.

\section{Résultats}

\section{Une vectorisation hétérogène}

Le test de vectorisation réalisé sur un secteur d'un kilomètre carré a mis en évidence une forte hétérogénéité dans la vectorisation des structures par divers opérateurs.

Cette hétérogénéité se marque (i) par des effectifs très différents du nombre d'objets reconnus, allant du simple au double, quel que soit la forme des objets enregistrés, (fig. 4) (ii) par des distributions spatiales des types de structures identifiées différentes. Nous avons donc cherché à déterminer les causes de cette hétérogénéité.

\section{L'effet des opérateurs}

Il semble exister une relation entre les effectifs enregistrés et les profils des opérateurs (fig. 4). Les plus faibles effectifs des objets enregistrés sous forme de point et de surface ont été enregistrés par un opérateur qui ne connait pas le milieu étudié (Opé. 6). De faibles effectifs ont également été observés pour un autre opérateur qui par contre, connait bien le terrain mais qui réalisait sa première expérience de vectorisation de données LiDAR (Opé. 3). Il semblerait qu'une bonne connaissance du milieu étudié et de l'outil de vectorisation soit nécessaire pour aboutir à une vectorisation la plus homogène possible.

\section{L'effet des indices d'imagerie}

Nous nous sommes également intéressés à observer si le choix de l'indice de visualisation utilisé avait une influence sur la reconnaissance du type de structure. L'ensemble des résultats obtenus a démontré que certains indices présentent un fort potentiel pour l'identification de certains types de structure (fig. 5). Comme par exemple la carte des pentes qui a permis d'identifier la majeure partie des charbonnières, des murées, des murées sur épaulements et des épaulements, ou bien encore le sky view factor utilisé pour reconnaitre les carrières et les zones de lapiaz. Nous avons pu voir que les charbonnières localisées en contexte de plateau n'ont pas pu être identifiées par le biais de la carte des pentes. Ainsi, l'utilisation d'un indice unique ne permet pas d'identifier l'ensemble des structures. Ceci confirme les observations faites par Challis et al. (2011) et Hesse (2010). De la même manière, nous avons observé que l'utilisation du sky view factor en contexte de fort relief (versant) ne permet pas d'identifier les structures présentes, comme il l'a déjà été démontré par Doneus (2013). Aussi l'utilisation d'indices non appropriés peut avoir une influence sur le nombre de structures identifiées.

\section{L'effet du respect du protocole}

Les résultats du test ont également mis en évidence que l'enregistrement des structures n'était pas réalisé de la même manière en fonction des opérateurs : les tertres allongés (fig. 6A, B et C), les carrières ou bien encore les zones de lapiaz ont été vectorisées selon plusieurs formes (fig. 6D, $\mathrm{E}$ et $\mathrm{F}$ ) ce qui induit (i) des effectifs différents, (ii) une surestimation et/ou une sous-estimation des longueurs et des surfaces cumulées (tabl. 2) ou bien encore (iii) masque la présence d'autres structures (fig. $6 \mathrm{~F}$ ). Ces observations montrent bien qu'il est nécessaire de définir et de respecter les règles de vectorisation pour aboutir à une vectorisation homogène. 

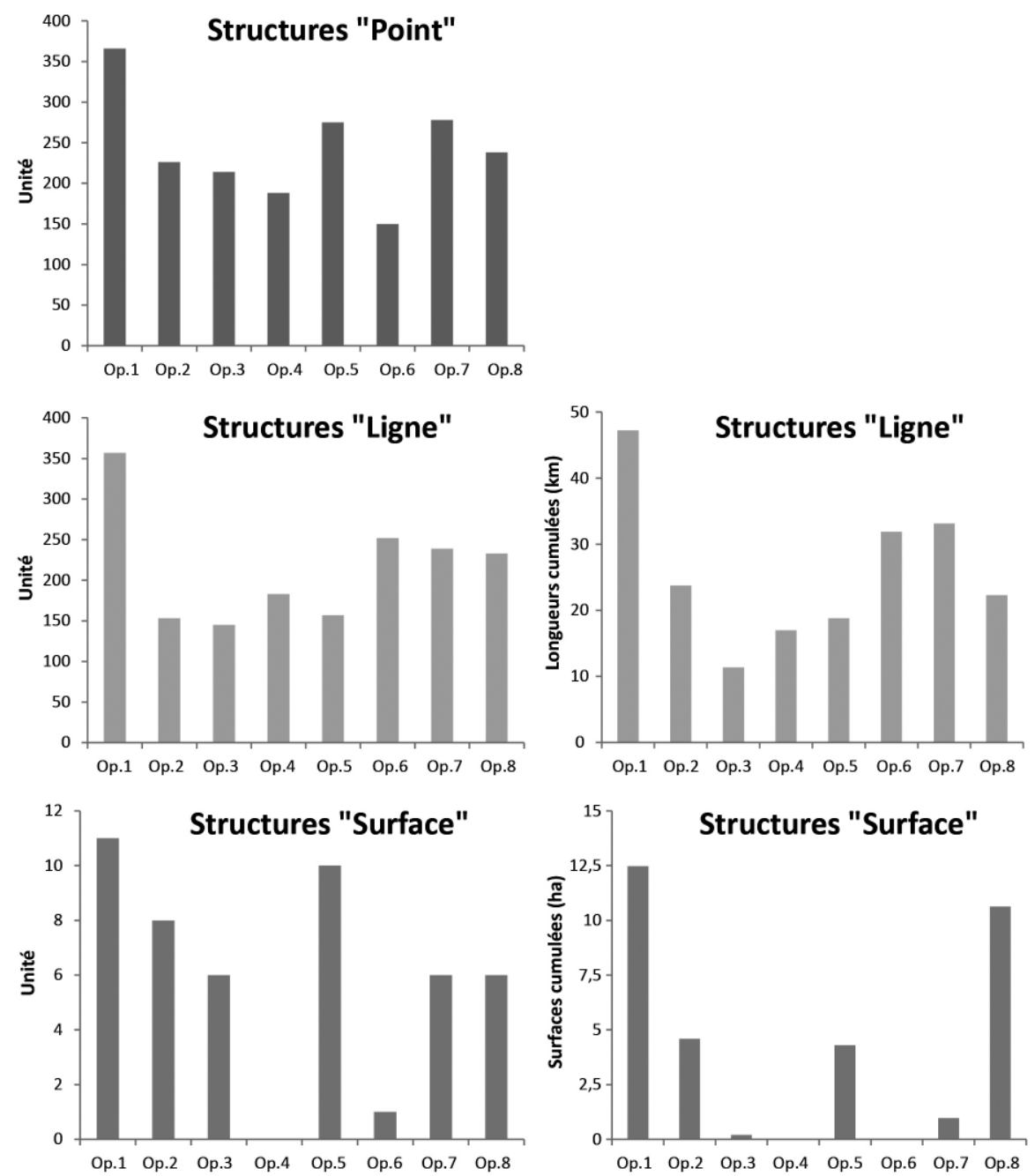

Figure 4 : Distribution des diverses entités spatiales identifiées selon chaque opérateur.

Figure 4 Distribution of spatial features identified by each operator.

Figure 5: (Voir planche couleur IX) Distribution des divers types d'indices de visualisation utilisés, par l'ensemble des opérateurs de saisie, en fonction du type de structure identifiée.

Figure 5: (See colour plate IX)

Visualization index distribution depen-

ding on each operator and remains.

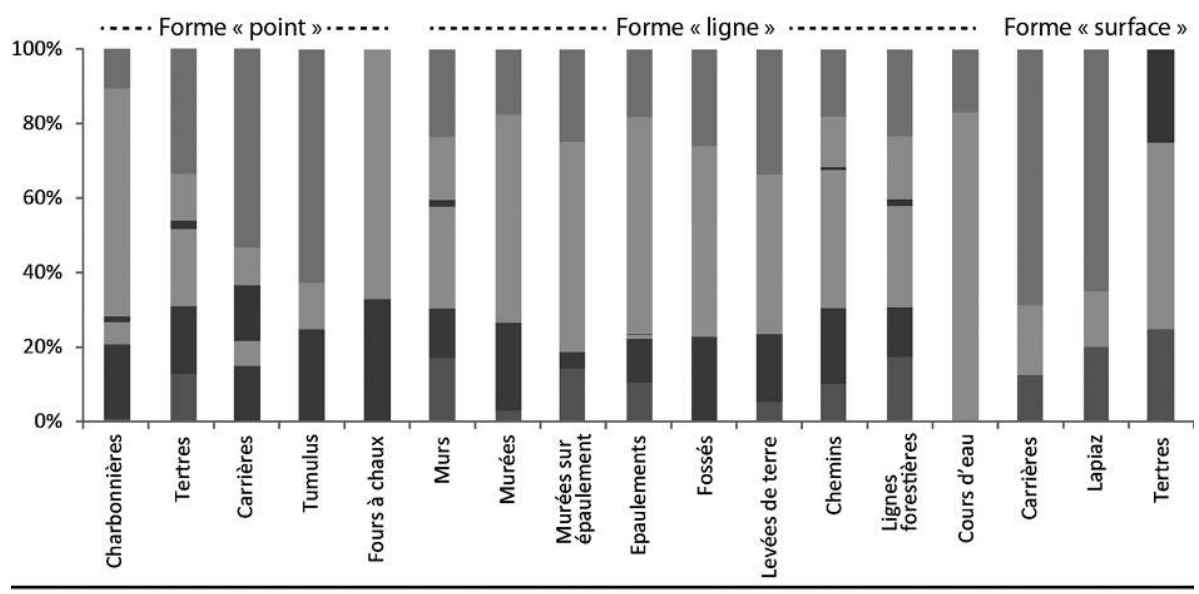

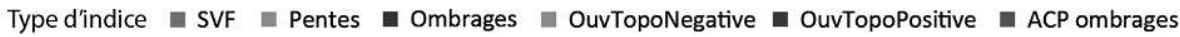


Figure 6 : (Voir planche couleur X) Enregistrement d'un tertre allongé $(\mathrm{A}, \mathrm{B}$ et $\mathrm{C})$ et d'une zone de lapiaz $(\mathrm{D}, \mathrm{E}$ et $\mathrm{F})$ selon divers formes i.e. point, ligne et surface.

Figure 6: (See colour plate X) Recording of a long barrow and a lapiaz area according to different features (point, line, polygon).
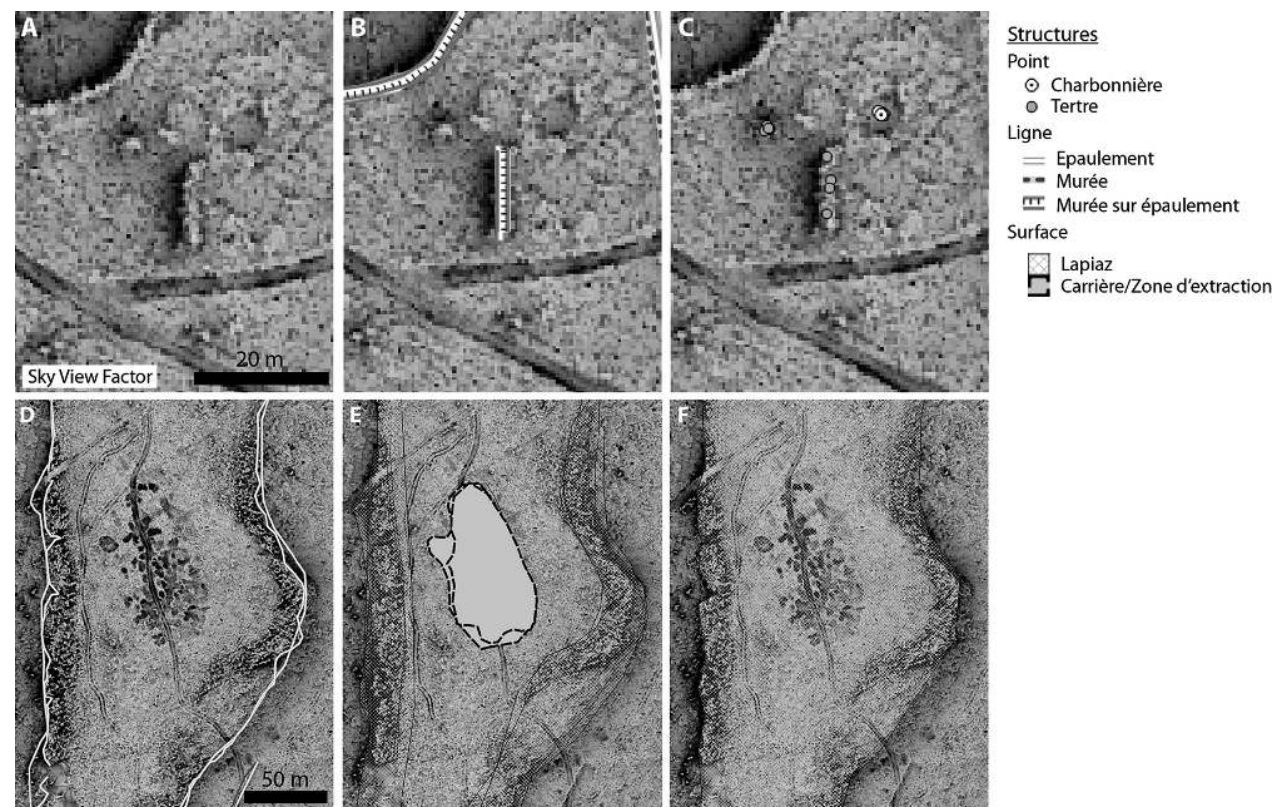

\begin{tabular}{|c|c|c|}
\hline Opérateur & Enregistrement & Surface enregistrée \\
\hline Opé. 1 & zone lapiaz & 5,2 ha \\
\hline Opé. 2 & zone lapiaz & 4,1 ha \\
\hline Opé. 5 & zone lapiaz & 3,9 ha \\
\hline Opé. 8 & zone « englobante » & 9,4 ha \\
\hline
\end{tabular}

au GNSS et à partir des données LiDAR. On peut supposer que les secteurs vides n'ont pas été prospectés au GNSS; que le relevé GNSS ne permet pas d'avoir une vision d'ensemble et donc une exhaustivité dans le relevé; ou bien que l'identification des structures sur le terrain n'était pas possible, soit du fait de la présence de végétation qui masquait les vestiges ou bien parce que ces derniers ne présentaient pas

Tableau 2: Différences des surfaces enregistrées pour un même lapiaz.

Table 2: Differences of recorded areas for the same lapiaz.

\section{Un enregistrement plus complet/exhaustif des vestiges}

Le secteur ayant été prospecté au GNSS au cours de la dernière décennie, les relevés ont ainsi pu être utilisés pour évaluer la reconnaissance des divers types de structures identifiées, par le géomaticien en charge du projet, à partir des données LiDAR. Il en ressort que les effectifs des structures relevés à partir des données LiDAR sont nettement supérieurs à ceux du GNSS (tabl. 3, fig. 7). Quelle que soit la forme des entités enregistrées, on observe une augmentation du nombre de relevés à partir du LiDAR supérieur à $50 \%$. Cette augmentation est du même ordre de grandeur que pour la forêt de Haye (Georges-Leroy et al., 2011) et pour toute la partie sud de la zone couverte par le LiDAR sur la forêt du Châtillonnais, couvrant $100 \mathrm{~km}^{2}$ (Goguey et al., 2014). Plusieurs hypothèses peuvent être formulées pour expliquer ces écarts entre le nombre de structures relevées seconde hypothèse a été validée par un retour terrain, où il a été observé que les structures de faibles amplitudes topographiques, de l'ordre de quelques décimètres, sont très difficilement repérables sur le terrain. De plus, certains vestiges relevés à partir du LiDAR n'étaient pas cartographiés lors des relevés GNSS, c'est le cas des charbonnières, qui sont hors du cadre chronologique des thématiques de recherches des chercheurs. Le relevé LiDAR sur cette zone permet de réaliser un inventaire plus complet des structures que la prospection GNSS.

\section{Une identification plus juste et plus précise des vestiges}

Quand on observe les images produites de données LiDAR, on peut très souvent, constater des anomalies topographiques, l'enjeu de telles données est surtout de pouvoir les identifier, les classer selon une typologie voire de les interpréter archéologiquement et historiquement. Outre la forte différence de structures enregistrées entre la lecture $\mathrm{LiDAR}$ et la prospection terrain, il existe également une hétérogénéité dans l'interprétation des vestiges, entre 


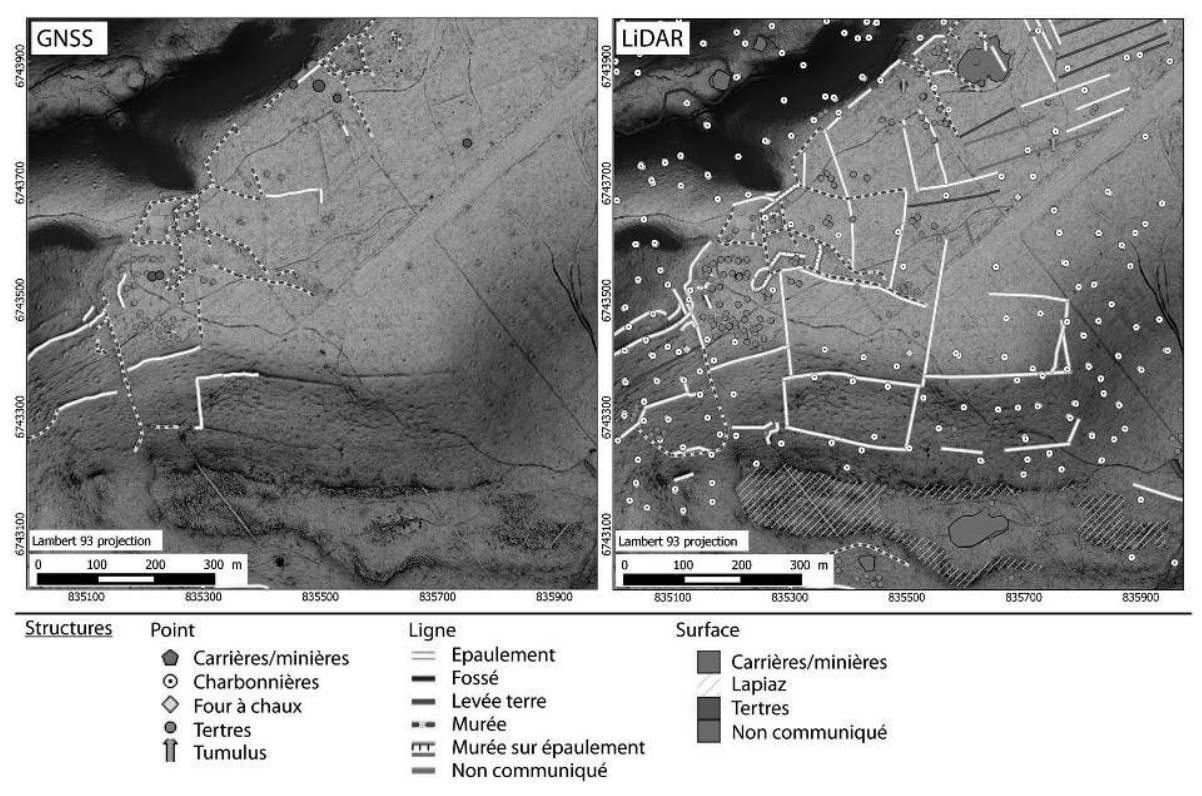

Figure 7 : (Voir planche couleur X) Cartographie de comparaison entre les structures archéologiques relevées au GNSS et celles identifiées par l'analyse des données LiDAR.

Figure 7: (See colour plate X) Comparison map between the archaeological structures surveyed by GNSS and those identified by LiDAR data analysis.

\begin{tabular}{|c|c|c|c|c|c|c|}
\hline \multirow{2}{*}{ Formes relevées } & LiDAR & GNSS & Différentiel & LiDAR & GNSS & Différentiel \\
\cline { 2 - 7 } & \multicolumn{2}{|c|}{ Unité } & LiDAR/GNSS & \multicolumn{2}{c|}{ Longueur cumulée $(\mathrm{km})$} & LiDAR/GNSS \\
\hline Point & 345 & 50 & $+299 \%$ & - & - & - \\
\hline Ligne & 140 & 65 & $+54 \%$ & 10,5 & 3,5 & $+100 \%$ \\
\hline
\end{tabular}

Tableau 3 : Comparaison des effectifs des structures relevées par prospection pédestre au GNSS et à partir des données LiDAR. Table 3: Vestiges amount comparison by GNSS surveys and by data LiDAR.

les relevés GNSS et LiDAR (fig. 7). Pour les entités enregistrées sous forme de point, les vestiges ont tous bien été identifiés. Pour ceux enregistrés sous forme de ligne, seulement $50 \%$ des épaulements relevés à partir du LiDAR correspondent à des épaulements vus en prospection GNSS (fig. 8A). Pour les murées, $90 \%$ de structures ont été bien classées. Par contre toutes les murées sur épaulement ont été mal classées (fig. 8A). Ceci peut s'expliquer par le fait, qu’à l'époque de l'acquisition GNSS, en 2003, les prospecteurs ne faisaient pas la différence entre les murées sur épaulements et les murées. Enfin, les fossés et les levées de terre identifiés à partir du LiDAR correspondent bien aux relevés GNSS. Pour les structures de formes « surface », l'ensemble des vestiges identifiés à partir du LiDAR présentent bien la même typologie que celle des relevés GNSS. Au vu de ces résultats, il apparait que l'identification du type de structure ne semble pas être aisée pour les formes linéaires. Toutefois, il est difficile de savoir si les erreurs de classification proviennent d'une mauvaise identification sur le terrain lors des relevés GNSS ou bien d'une mauvaise interprétation des indices de visualisation issus des relevés LiDAR ou bien encore d'un problème de typologie.
La typologie des structures archéologiques de la forêt du Châtillonnais étant en partie définie à partir des caractéristiques morphologiques de celles-ci, l'utilisation de coupes topographiques $2 \mathrm{D}$ semble être adaptée à la reconnaissance des types de vestiges. Les linéaires qui présentaient des différences typologiques entre les relevés LiDAR et GNSS ont donc été vérifiés à partir de ces coupes $2 \mathrm{D}$ réalisées sur le MNT LiDAR. Les résultats obtenus montrent que les structures bien classées sont plus nombreuses par rapport au GNSS. Ainsi, les épaulements et les murées ont bien été identifiés à hauteur de $80 \%$ (fig. 8B). Plus de $2 / 3$ des murées sur épaulement ont été reconnues. Toutefois, l'utilisation des coupes topographiques $2 \mathrm{D}$ pour évaluer la bonne reconnaissance des structures à partir des indices de visualisation reste limitée. Dans certains cas, il est très difficile à partir des coupes 2D d'identifier avec certitude le type et plus particulièrement pour les murées sur épaulements et pour les levées de terre (fig. 8B). Il semble donc que la haute résolution des données LiDAR permette de mieux identifier les types de structures que lors des relevés GNSS. Les données LiDAR permettent d'identifier les fines variations topographiques qui ne sont pas forcément visibles sur le 
Figure 8 : (Voir planche couleur XI) Histogrammes illustrant la reconnaissance des types de structure identifiés à partir du LiDAR par rapport aux relevés GNSS (A) et aux coupes topographiques 2D (B). Figure 8: (See colour plate XI) Type of remains identification histograms from the LiDAR compared with GNSS surveys (A) and compared with the $2 D$ topographic cross sections $(B)$.

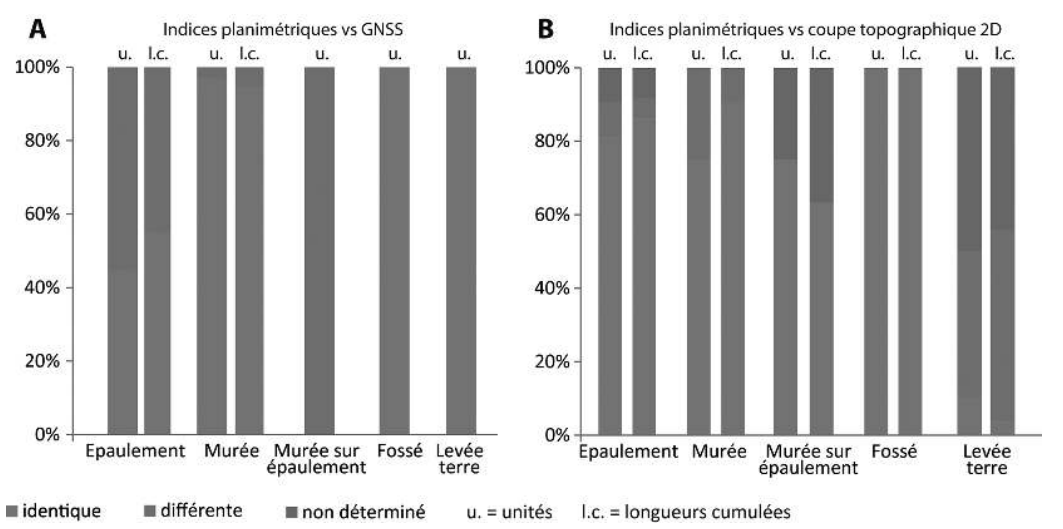

terrain. De plus, lorsque les relevés GNSS ont été réalisés, les changements typologiques lors de l'enregistrement d'une structure n'ont pas forcément été pris en compte sur le terrain et dépendent donc de l'opérateur.

L'utilisation des relevés GNSS et des coupes topographiques $2 \mathrm{D}$ réalisées sur le MNT LiDAR offre la possibilité d'effectuer une validation partielle du type de vestige identifiée. Il semble donc nécessaire d'effectuer un retour sur le terrain pour valider l'identification des vestiges linéaires qui présentent de faibles amplitudes topographiques.

\section{Conclusion}

Le protocole de vectorisation proposé a pu être testé sur un secteur de la forêt du Châtillonnais par divers opérateurs. Du point de vue pratique, celui-ci permet à chacun d'utiliser un outil commun, des règles de vectorisation communes, facilitant l'enregistrement des données, notamment pour les novices en logiciel SIG. De plus, ce protocole vise à aboutir à une vectorisation la plus homogène possible de l'ensemble des vestiges de la forêt du Châtillonnais.

Sur le secteur d'étude, nous avons aussi pu mettre en évidence que certains indices de visualisation présentaient un fort potentiel pour l'identification typologique des structures comme celui de la carte des pentes. Toutefois, l'utilisation d'un indice unique ne permet pas l'identification de toutes les structures présentent sur le secteur, confirmant ainsi les observations faites par Challis et al. (2011) et Hesse (2010). Nous avons essayé de synthétiser dans le tableau 4 les associations entre les types de structures morphologiques, les indices et les contextes géomorphologiques. Naturellement ce tableau ne prend pas en compte l'importance (hauteur ou profondeur) du vestige conservé. Les résultats obtenus lors du test de vectorisation nous ont permis d'améliorer le protocole de vectorisation en plusieurs points. Des règles plus précises ont été définies pour homogénéiser l'enregistrement (1). En fonction du type de structure et du contexte géomorphologique, les indices les plus adaptés ont été identifiés (2). L'utilisation des coupes topographiques en 2D permet aux opérateurs, dans la majeure partie des cas, de valider l'identification typologique réalisée à partir des indices de visualisation (3).

Pour les vestiges de la forêt du Châtillonnais, il a été démontré que les données LiDAR permettaient d'identifier $50 \%$ de structures archéologiques en plus par rapport aux relevés GNSS effectués sur le terrain. La haute résolution spatiale des données LiDAR permet une visualisation plus précise des formes des structures par rapport au terrain; elle facilite ainsi l'identification typologique des vestiges archéologiques. Toutefois, pour les linéaires qui présentent de faibles amplitudes topographiques, l'identification des types de structures à partir des données LiDAR reste dans certains cas difficilement réalisable, rendant nécessaire la validation des données obtenues par des retours sur le terrain.

\section{Remerciements}

Nous tenons à remercier le GIP du futur parc national des forêts de Champagne et de Bourgogne pour la mise à disposition des données $L i D A R$, son soutien financier et le dialogue constant pour mener à bien ces travaux et le Conseil Régional de Bourgogne qui a financé ces travaux (PARI 2013, 2014). 


\begin{tabular}{|c|c|c|c|c|c|c|}
\hline Types/indices & SkyView Factor & Pentes & $\begin{array}{l}\text { Ouv. Topo posi- } \\
\text { tive }\end{array}$ & Ouv. Top négative & ACP Ombrage & Ombrage \\
\hline Carrières & Plateau & & & & & $\begin{array}{l}\text { Plateau/ } \\
\text { Versant }\end{array}$ \\
\hline Charbonnières & & Versant & $\begin{array}{c}\text { Plateau/ } \\
\text { Fond de vallon }\end{array}$ & & & \\
\hline Chemins & Plateau & Plateau & $\begin{array}{c}\text { Plateau/ } \\
\text { Versant/ Fond de } \\
\text { vallon }\end{array}$ & $\begin{array}{c}\text { Plateau/ } \\
\text { Versant/ Fond de } \\
\text { vallon }\end{array}$ & & \\
\hline Fossé & Plateau & Plateau & $\begin{array}{c}\text { Plateau/ } \\
\text { Versant/ Fond de } \\
\text { vallon }\end{array}$ & $\begin{array}{c}\text { Plateau/ } \\
\text { Versant/ Fond de } \\
\text { vallon }\end{array}$ & & \\
\hline Four à Chaux & & Versant / Plateau & Versant / Plateau & & & \\
\hline Lapiaz & Plateau & & & & $\begin{array}{l}\text { Plateau/ } \\
\text { Versant }\end{array}$ & \\
\hline Murée/Levée & Plateau & Plateau & Plateau/ Versant & & & \\
\hline $\begin{array}{l}\text { Murée sur épau- } \\
\text { lement }\end{array}$ & Plateau & Plateau & $\begin{array}{l}\text { Plateau/ } \\
\text { Versant }\end{array}$ & & $\begin{array}{l}\text { Plateau/ } \\
\text { Versant }\end{array}$ & \\
\hline Epaulement & & Plateau & & & Plateau & Plateau \\
\hline $\begin{array}{c}\text { Tertres / Pierriers / } \\
\text { Tumulus }\end{array}$ & Versant / Plateau & & Versant / Plateau & Versant / Plateau & Plateau/ Versant & Plateau/Versant \\
\hline
\end{tabular}

Tableau 4 : Association des indices et des types de forme; il est indiqué pour chaque type de structure l'indice à utiliser en fonction des contextes géomorphologiques (plateau, versant, fond de vallon).

Table 4: Association between visualization indexes and archaeological features: for each type of remains the best indexes to be used according to geomorphological context (ridgetop, valley slope, and valley floor) are indicated.

\section{Bibliographie}

Bofinger, J. et Hesse, R., 2010. Der Einsatz von Airborne Laserscanning zur Entdeckung von archäologischen Geländedenkmalen. In: Bofinger, J. and M. Merkl (Eds.): Mit Hightech auf den Spuren der Kelten. Archäologische Informationen aus Baden-Württemberg 61, p. 70-89.

Challis, K., Forlin, P., Kincey, M., 2011. A generic toolkit for the visualization of archeological features on airborn LiDAR elevation data. Archeological Prospection, 18, p. 279-289.

Chase, A. F., Chase, D.Z., Weishampel, J.F. 2010. Lasers in the Jungle. Airborne sensors reveal a vast Maya landscape. Archaeology, 63 (4), p. 27-29.

Chaume, B., Mordant, C., 2011. Le complexe aristocratique de Vix. Nouvelles recherches sur l'habitat, le système de fortification et l'environnement du mont Lassois. Ed. Universitaires de Dijon, 2 vol. (Art, archeology et patrimoine).

Devereux, B.J., Amable, G.S., Crow, P., 2008. Visualization of LiDAR terrain models for archaeological feature detection. Antiquity, 82, p. 470-479.

Devereux, B.J., Amable, G.S., Crow, P., Cliff, A.D. 2005. The potential of airborne lidar for detection of archaeological features under woodland canopies. Antiquity, 79, p. 648-660.
Doneus, M., 2013. Openness as Visualization Technique for Interpretative Mapping of Airborne Lidar Derived Digital Terrain Models. Remote sensing, 5 (12), p. 6426-6442.

Doneus, M., Briese, C., 2006. Digital terrain modelling for archaeological interpretation within forested areas using full-waveform laserscanning. In: M. Ioannides, D. Arnold, F. Niccolucci und K. Mania (Editors), The 7th International Symposium on Virtual Reality, Archaeology and Cultural Heritage, VAST, p. 155-162.

Dupouey J.-L., Dambrine E., Dardignac C., Georges-Leroy M., éd. 2007. La mémoire des forêts. Actes du colloque "Forêt, Archéologie et Environnement " 14-16 décembre 2004, ONF INRA - DRAC Lorraine, 294 p.

Evans, D., 2016. Airborne laser scanning as a method for exploring long-term socio-ecological dynamics in Cambodia. Journal of Archaeological Science, 74, p. 164-175.

Fruchart, C., 2014. Analyse spatiale et temporelle des paysages de la forêt de Chailluz (Besançon, Doubs) de l'Antiquité à nos jours. Thèse de doctorat, Université de Franche-Comté, France.

Georges-Leroy, M., Bock, J., Dambrine, E., Dupouey, J.L., 2011. Apport du Lidar à la connaissance de l'histoire de l'occupation du sol en forêt de Haye. Archeosciences, 35, p. 117-129. 
Georges-Leroy, M., Bock, J., Dambrine, E., Dupouey, J.L., 2009. Le massif forestier, objet pertinent pour la recherche archéologique. L'exemple du massif forestier de Haye (Meurthe-etMoselle). Revue Géographique de l'Est [En ligne], vol. 49 / 2-3, mis en ligne le 01 avril 2013. URL : http://rge.revues.org/1931

Goguey, D., Pautrat, Y., Guillaumet, J.P., Thevenot, J.P., Popovitch, L., et al., 2010. Dix ans d'archéologie forestière dans le Châtillonnais (Côte-d'Or) : enclos, habitats, parcellaires. Revue archéologique de l'Est, Société archéologique de l'Est, 59(1), p. 99-209.

Goguey, D., Cordier, A., Chevigny, E., 2014. Typologie des enclos dans les forêts du Châtillonnais (Côte-d'Or) : Enclos d'habitats et enclos cultuels. Séminaire Workshop 2 Les parcellaires conservés sous fôret, May 2014, Paris, France.

Hesse, R., 2010. LiDAR-derived Local Relief Models - a new tool for archaeological prospection. Archaeological Prospection, $17(2)$, p. $67-72$

Jones, K.H., 1998. A comparison of algorithms used to compute hill slope as a property of the DEM. Computers \& Geosciences, 24(4), p. 315-323.

Kokalj, Ž., Zakšek, K., Oštir, K., 2011. Application of Sky-View Factor for the Visualization of Historic Landscape Features in Lidar-Derived Relief Models. Antiquity, 85, p. 263-273.

Kooistra, M. J. et Maas, G. J., 2008. The widespread occurrence of Celtic field systems in the central part of the
Netherlands. Journal of Archaeological Science, 35, p. 23182328.

Nuninger, L., Fruchart, C., Opitz, R., avec la collaboration de l'équipe Lieppec et de l'association ARESAC, 2010. Lidar : quel apport pour l'analyse des paysages? Bulletin AGER, p. 34-43.

Paris, R., Bretonne, C., 1981. Répertoire des sites gallo-romains du massif forestier de Châtillon-sur-Seine, Société Archéologique et Historique du Châtillonnais, inédit.

Sittler B., Hauger, K., 2007). Les apports du laser aéroporté à la documentation de parcellaires anciens fossilisés par la forêt : l'exemple des champs bombés de Rastatt en Pays de Bade. In Dupouey et al. 2007, p. 155-161.

Stular, B., Kokalj, Z., Ostir, K, Nuninger, L., 2012. Visualization of lidar-derived relief models for detection of archeological features. Journal of Archeological Science, 39(11), p. 3354- 3360.

Yokoyama, R., Shirasawa, M., Pike, R. J., 2002. Visualizing topography by openness: A new application of image processing to digital elevation models. Photogrammetric Engineering and Remote Sensing, 68, p. 251-266.

Zaksek, K., Ostir, K., Kokalj, S., 2011. Sky-View Factor as a Relief Visualization Technique. Remote Sensing, 3, p. 398-415. 